\title{
Réduction des charges alimentaires des cobayes (Cavia porcellus L.) par un régime fourrager supplémenté par la farine de poisson et/ou du maïs-grain durant le cycle de reproduction
}

\author{
N'Goran David Vincent KOUAKOU* et Yao Brice Savier BROU \\ Département de Formation et de Recherche Agriculture et Ressources animales, Institut National \\ Polytechnique Félix Houphouët-Boigny, B.P. 1313 Yamoussoukro, Côte d'Ivoire. \\ *Auteur correspondant ; E-mail: kwayki@yahoo.fr; Tél : +225 08393 363,
}

\section{RESUME}

La supplémentation de Panicum maximum Jacq par l'aliment concentré granulé pour lapin a l'avantage d'induire une meilleure productivité des cobayes (Cavia porcellus L.) et a pour inconvénient d'être relativement coûteux. Afin de réduire le coût de production des cobayes, quatre régimes alimentaires que sont : Panicum maximum et Euphorbia heterophylla associé au maïs-grain (PEM), ou associé à la farine de poisson (PEF), ou associé au grain de maïs et à la farine de poisson (PEMF) et Panicum maximum associé au granulé pour lapin (PG; régime témoin), ont été distribués ad libitum à 24 cobayes femelles multipares durant la gestation et l'allaitement. Les valeurs moyennes du taux de fécondité, des poids à la naissance et des croissances journalières des cobayes au cours de la lactation ont été $83,100,167$ et $200 \% ; 71 \pm 14,88 \pm 18,98 \pm 17$ et $104 \pm 10$ $\mathrm{g}$; et $5 \pm 1,6 \pm 1,7 \pm 1$ et $8 \pm 1 \mathrm{~g} . \mathrm{j}^{-1}$ respectivement pour PEM, PEF, PEMF et PG. Les résultats obtenus dans cette étude montrent que le régime constitué de Panicum maximum, Euphorbia heterophylla et de farine de poisson (PEF) semble être une solution optimale en milieu rural, avec une réduction de 55\% des charges liées à l'alimentation en comparaison à PG

(C) 2016 International Formulae Group. All rights reserved.

Mots clés : Cobaye, fourrage, Euphorbia heterophylla, Panicum maximum, farine de poisson, économie.

\section{Reduction of guinea pig food costs (Cavia porcellus L.) by distribution of fodders supplemented by fish meal or/and corn kernels during the reproductive cycle}

\begin{abstract}
Supplementing Panicum maximum Jacq. with pelleted rabbit concentrate feed has the advantage of inducing better productivity in domestic cavies, but having the disadvantage of being relatively expensive. In order to reduce feed expenses for the production of cavy carcass, four diets: Panicum maximum and Euphorbia heterophylla $\mathrm{L}$. associated with maize kernels (PEM), or associated with fish meal (PEF), or associated with a mix of fish meal and maize kernels (PEMF); and P. maximum associated with rabbit pellets (PG; control diet),
\end{abstract}


were distributed ad libitum to 24 multiparous female cavies during gestation and lactation. The rates of fecundity, individual birth weight, and weight gain during lactation of the kids were 83, 100, 167 and 200\%; $71 \pm 14,88 \pm 18,98 \pm 17$ and $104 \pm 10 \mathrm{~g}$; and $5 \pm 1,6 \pm 1,7 \pm 1$ and $8 \pm 1$ g.d ${ }^{-1}$, respectively, for PEM, PEF, PEMF and PG diets. Results of this study indicated that P. maximum and E. heterophylla supplemented with fish meal (PEF) could be an optimal solution in rural areas with a reduction of 55\% of feed expenses compared to PG.

(C) 2016 International Formulae Group. All rights reserved.

Keywords: Guinea pig, fodder, Euphorbia heterophylla, Panicum maximum, fish meal, economy.

\section{INTRODUCTION}

En Côte d'Ivoire, l'un des meilleurs régimes alimentaires pour cobaye (Cavia porcellus L.) est un régime mixte constitué de l'aliment concentré granulé pour lapin et de Panicum maximum Jacq (Kouakou et al., 2015a). S'il a l'avantage d'induire une meilleure productivité (taux de fécondité, taille de portée, poids à la naissance et croissance journalière) chez les cobayes, il a pour inconvénient d'être relativement coûteux, amenant ainsi les caviaculteurs à se tourner vers les plantes fourragères telles que Tithonia diversifolia (Hemsl.) A. Gray et Pennisetum purpuruem (Noumbissi et al., 2014), Euphorbia heterophylla, Ipomoea batatas et Panicum maximum (Bindelle et al., 2007; Kouakou et al., 2012; Kouakou et al., 2015b), Arachis glabarta, Desmodium intortum et Centrosoma pubescens (Tchoumboué et al., 2001), Moringa oleifera (Tedonkeng et al., 2005), ou des tubercules comme le manioc (Manihot esculenta Crantz) (Mweugang et al., 2016), etc. Toutefois, les régimes fourragers demeurent inefficients en raison de leur encombrement qui limite la quantité d'énergie retirée par l'animal (Baumont et al., 2009).

Afin d'y remédier, certains auteurs suggèrent d'une part, la nécessité d'augmenter la densité énergétique de ces régimes fourragers à partir d'aliment tel que le maïsgrain (Bindelle et al., 2009; Yapi et al., 2013), et d'autre part, une complémentation au minimum de $2 \%$ de protéines d'origine animale pour couvrir les besoins du cobaye en acide aminés (méthionine : $0,39 \%$ de la ration, cystine : $0,03 \%$, tryptophane : 0,16 à $0,25 \%$, arginine : 0,3\%) (Fuss, 2002). Dans le but de permettre aux populations démunies de disposer de source de protéines animales de bonne qualité nutritionnelle grâce à la production des cobayes (Cavia porcellus L.) à moindre coût, divers régimes alimentaires alternatifs à la distribution du régime composé de Panicum maximum Jacq et de concentré granulé pour lapin ont été étudiés. L'objectif de l'étude est de comparer l'aliment concentré granulé pour lapin associé à Panicum maximum à un régime fourrager (Panicum maximum et Euphorbia heterophylla) supplémenté par la farine de poisson et/ou du maïs-grain durant le cycle de reproduction des cobayes.

\section{MATERIEL ET METHODES}

L'étude a été conduite d'août à décembre 2010, à la ferme expérimentale de l'Institut National Polytechnique Félix Houphouët-Boigny (INP-HB) de Yamoussoukro (Côte d'Ivoire) $\left(6,5^{\circ} \mathrm{N}\right.$. ; $5,2^{\circ} \mathrm{O}$.). Durant l'essai, la température moyenne et l'humidité relative de la région ont été respectivement de $29 \pm 1{ }^{\circ} \mathrm{C}$ et de $81 \pm 1 \%$. La pluviométrie moyenne mensuelle était de $950 \mathrm{~mm}$.

\section{Dispositif expérimental}

Douze mâles âgés de 15 à 20 mois, ayant un poids moyen de $710,4 \pm 09,6 \mathrm{~g}$ et dont la fertilité avait été vérifiée antérieurement avec d'autres femelles et 24 femelles multipares non gravides âgées de huit mois et d'un poids moyen de $600,5 \pm 35,3 \mathrm{~g}$, ont été repartis aléatoirement par lot de trois cobayes (deux femelles et un mâle) par loge grillagée de $40 \mathrm{dm}^{3}$. Tous les animaux en bon état sanitaire, étaient du type «à poils ras \& dessin », possédant une variété de robes dominées par trois couleurs (marron, blanc et noir). Les aliments expérimentaux étaient 
constitués de fourrages, de grains de maïs, de farine de poisson et de granulé pour lapin. Les fourrages Panicum maximum au stade épiaison et Euphorbia heterophylla au stade floraison, ont été tous deux récoltés dans le périmètre de l'INP-HB et distribués aux animaux quotidiennement sous forme de feuilles et de tiges fraîches. Les graines de Zea mays ou maïs-grain jaune (Poaceae), la farine de poisson commerciale (Morroco) et le granulé pour lapin de $3 \mathrm{~mm}$ de diamètre (SIPRA-IVOGRAIN, Abidjan, Côte d'Ivoire) ont été achetés dans le commerce. Quatre régimes expérimentaux ont été constitués : Panicum maximum et Euphorbia heterophylla associé au maïs-grain (PEM) ; Panicum maximum et Euphorbia heterophylla associé à la farine de poisson (PEF) ; Panicum maximum et Euphorbia heterophylla associé au maïs-grain et à la farine de poisson (PEMF) et Panicum maximum associé au granulé pour lapin (PG) (Régime témoin). Chaque régime a été distribué ad libitum et répété sur trois lots de cobayes. Les quantités de Euphorbia heterophylla et de Panicum maximum distribuées étaient respectivement $250 \mathrm{~g}$ et $500 \mathrm{~g}$ de matières fraîches par animal. Celles de la farine de poisson, du granulé pour lapin et du maïs-grain distribuées, étaient respectivement 5, 40, $50 \mathrm{~g}$ de produit brut par animal.

\section{Conduite de l'essai}

L'essai a duré 122 jours (4 mois) reparti en trois périodes. Il a débuté par une période d'adaptation aux régimes expérimentaux de 14 jours, avant la mise au mâle. La période d'accouplement et de gestation de 70 à 87 jours a débuté par l'introduction d'un mâle au sein de chaque groupe de femelles, durant 17 jours correspondant à un cycle œstral. A l'approche des mises bas, toutes les femelles ont été placées en cage individuelle de maternité de $40 \mathrm{dm}^{3}$, jusqu'à la fin de la période de lactation de 21 jours. Au cours de la lactation, les femelles et leurs portées ont été alimentées ensemble. Les aliments et l'eau de boisson ont été distribués deux fois par jour. Les aliments distribués et les refus d'aliments ont été pesés régulièrement avant chaque nouvelle distribution. Les animaux ont reçu chaque semaine une orange récoltée dans le périmètre de l'INP-HB comme apport en vitamine C. Durant l'essai, les femelles à jeun, étaient pesées chaque semaine. Le nettoyage des cages et du bâtiment d'élevage a été quotidien. $\mathrm{Au}$ cours de l'essai, des échantillons des aliments expérimentaux ont été prélevés pour déterminer leurs compositions chimiques (matière sèche, matière organique, matière azotée totale et fibre brute) telle que recommandé par l'A.O.A.C. (2006).

\section{Analyses statistiques}

A la fin de l'étude, les valeurs moyennes par régime de l'ingestion volontaire journalière de la matière sèche, du gain moyen quotidien (GMQ) des animaux durant les périodes de gestation et de lactation, de même que celles de certains paramètres de reproduction ont été déterminées. Elles ont été soumises à l'analyse de variance (ANOVA) à un facteur en considérant la taille de la portée comme covariable au seuil de signification de $5 \%$ à l'aide du logiciel STATA 12.0 (STATA, 2012). Le calcul des charges alimentaires pour la production d'un kilogramme de poids vif de cobaye a été fait à partir des hypothèses suivantes :

- Le revenu journalier d'un ouvrier agricole qui est de $2000 \mathrm{~F} \mathrm{CFA}$ pour $8 \mathrm{~h}$ de travail en Côte d'Ivoire. La recherche des aires de collecte et la récolte de $30 \mathrm{~kg}$ de fourrages (Euphorbia heterophylla et Panicum maximum) par un ouvrier agricole s'effectuent en 2 heures en moyenne ;

- Le coût du kilogramme du concentré granulé pour lapin IVOGRAIN-SIPRA, était à 250 F CFA (19/06/2010), celui de la farine de poisson était de $650 \mathrm{~F}$ CFA. Quant à celui du maïs-grain, il était de 120 F CFA.

\section{RESULTATS}

La composition chimique des aliments expérimentaux et les estimations des énergies métabolisables sont présentées dans le Tableau 1. Au cours de l'essai, les animaux n'ont présenté aucun trouble de santé et aucun 
cas de mortalité n'a été enregistré.

\section{Période de gestation}

Les valeurs moyennes de l'ingestion journalière de la matière sèche (IVJMS) des femelles soumises à $\mathrm{PG}$ différaient de celles des autres régimes $(\mathrm{P}<0,05)$, qui elles, ne différaient pas durant la gestation $(\mathrm{P}>0,05)$ (Tableau 2). Toutefois, chez ces derniers, le niveau d'IVJMS de Panicum maximum variait significativement avec l'apport de la farine de poisson. Quant au niveau d'ingestion de l'IVJMS de Euphorbia heterophylla, il différait selon l'apport du maïs-grain. En effet, l'IVJMS de Panicum maximum était plus faible chez PEM comparativement à PEMF ou à PEF $(\mathrm{P}<0,05)$. Quant à l'IVJMS de Euphorbia heterophylla, elle était plus élevée chez PEF comparativement à PEM ou PEMF $(\mathrm{P}<0,05)$. Les coefficients d'utilisation digestive apparent (CUDa) de la matière sèche et de la matière organique de PEM étaient significativement supérieurs à ceux des autres régimes $(\mathrm{P}<0,05)$ (Tableau 3). A l'inverse, le CUDa de la matière azotée totale de PEM étaient significativement inférieur a ceux des autres régimes $(\mathrm{P}<0,05)$. Les plus faibles digestibilités étant enregistrées au niveau de PG. Les tailles moyennes et les poids de portée ne différaient pas selon les régimes alimentaires $(\mathrm{P}>0,05)$. Les poids moyens avant les mises-bas des reproductrices soumises à $\mathrm{PG}$, différaient de ceux des autres reproductrices $(\mathrm{P}<0,05)$, qui eux, ne différaient pas $(\mathrm{P}>0,05)$. Le taux de fécondité des femelles nourries à $\mathrm{PG}$ était supérieur à ceux des autres femelles. Les poids moyens des cochonnets à la naissance sous PEM ont été significativement inférieurs à ceux de PEF et PG qui eux-mêmes n'étaient pas significativement différents. Quant aux poids moyens à la naissance des cochonnets sous
PEMF, ils ne différaient pas de ceux des trois autres régimes $(\mathrm{P}>0,05)$.

\section{Période de lactation}

Les valeurs moyennes de l'ingestion journalière de la matière sèche (IVJMS) des femelles soumises à $\mathrm{PG}$ différaient de celles des femelles sous PEM $(\mathrm{P}<0,05)$. Les valeurs moyennes de l'IVJMS des femelles soumises chacun de ces deux régimes ne différaient pas de celles des femelles sous les autres régimes (P>0,05). Le niveau d'IVJMS de Panicum maximum sous $\mathrm{PG}$, différait significativement de ceux des autres régimes, chez qui les niveaux d'IVJMS de ce fourrage, ne variaient pas malgré l'apport de la farine de poisson, de Euphorbia heterophylla, ou de maïs-grain. L'IVJMS de Euphorbia heterophylla, est restée plus faible chez PEM comparativement aux régimes ayant reçu la farine de poisson (PEF et PEMF) $(\mathrm{P}<0,05)$. Au sevrage, les poids moyens, de même que les croissances journalières des jeunes cobayes soumises à PG différaient de ceux des sevrons sous PEM $(\mathrm{P}<0,05)$. Ces paramètres de croissance chez les sevrons sous PEMF et PEF ne différaient pas de ceux des sevrons sous PEM et PG $(\mathrm{P}>0,05)$. Les pertes de poids des femelles reproductrices ne différaient pas non plus entre les régimes $(\mathrm{P}>0,05)$ (Tableau 4).

L'analyse de la relation coût relatif des aliments expérimentaux ingérés par femelle suitée au cours du cycle de reproduction et gain de poids résultant, a montré que PEMF induit un gain de poids meilleur pour un coût plus faible lorsque les coûts d'acquisition et de distributions des fourrages Panicum maximum et Euphorbia heterophylla sont pris en compte. Cependant, cette prise en compte des charges liées aux fourrages n'étant pas toujours vrai dans les milieux ruraux, le régime PEF s'avère alors plus économique par rapport au gain de poids obtenu (Tableau 5). 
Tableau 1: Compositions chimiques des aliments utilisés dans les régimes étudiés au cours de l'essai.

\begin{tabular}{|c|c|c|c|c|c|}
\hline $\begin{array}{l}\text { Composition chimique } \\
\text { (\% MS) }\end{array}$ & $\begin{array}{l}\text { Panicum } \\
\text { maximum }\end{array}$ & $\begin{array}{c}\text { Euphorbia } \\
\text { heterophylla }\end{array}$ & $\begin{array}{c}\text { Granulé } \\
\text { pour lapin }\end{array}$ & $\begin{array}{l}\text { Farine de } \\
\text { poisson }\end{array}$ & Maïs \\
\hline Matière sèche* & $21,0 \pm 3,4$ & $14,4 \pm 2,5$ & $91,5 \pm 1,8$ & 90,1 & 86 \\
\hline Matière organique & $90,8 \pm 1,6$ & $87,8 \pm 0,9$ & $90,1 \pm 1,1$ & 66,9 & 84,9 \\
\hline Fibres brutes & $32,1 \pm 1,5$ & $20,5 \pm 0,3$ & $14,6 \pm 3,6$ & 0 & 2,7 \\
\hline Matière azotée totale & $9,6 \pm 0,9$ & $16,5 \pm 1,2$ & $13 \pm 0,5$ & 53,8 & 10,3 \\
\hline Matière grasse & $2,5 \pm 0,5$ & $7,2 \pm 1,1$ & $3,3 \pm 1,2$ & 7,1 & 4,4 \\
\hline $\begin{array}{l}\text { Energie métabolisable } \\
\left(\mathrm{Kj}^{\mathrm{kg}} \mathrm{kg}^{-1} \mathrm{MS}\right)^{* *}\end{array}$ & 3618,3 & 8483,2 & 10178,6 & 12502,6 & 13959,4 \\
\hline
\end{tabular}

\section{DISCUSSION}

Les performances zootechniques et économiques enregistrées par le régime PG confirment bien les hypothèses initiales. Ces résultats sont à mettre au compte du mode de l'amélioration de la densité énergétique de la ration, de la qualité nutritionnelle de la ration notamment avec la présence de la farine de poisson dans les matières premières constitutives du granulé pour lapin et à la présence de Panicum maximum comme source de cellulose (Kouakou et al., 2015; Miégoué et al., 2016). En effet, le granulé pour lapin est un aliment industriel complet qui distribué aux cochons d'inde, doit être absolument supplémenté de fourrage grossier compte tenu de leurs besoins en cellulose plus importants et de la spécificité de leur physiologie digestive qui leur permet de mieux digérer les fibres (Sakaguchi et al., 1997). Les raisons à cette situation sont liées à une meilleure capacité de fermentation basée notamment sur la non rétention sélective des fluides et des particules du digesta, de même que le refoulement (reflux sélectif des microorganismes) des bactéries piégées dans le côlon vers le cæcum (Sakaguchi, 2003; Franz et al., 2011).
Cependant, de telles performances zootechniques reviennent relativement coûteuses lorsque l'on compare les coûts de l'alimentation relatifs pour $100 \mathrm{~g}$ de gain de poids des femelles suitées (Tableau 5). Aussi, les solutions alternatives de production à moindre coût s'avèrent utiles. Des trois régimes alternatifs au régime témoin $(\mathrm{PG})$, PEM paraît être le plus simple à obtenir en milieu rural. Malheureusement, il induit des performances médiocres. Plusieurs raisons pourraient justifier cet état de fait : le déséquilibre énergie/protéine important du régime induit par l'apport du maïs-grain, un aliment énergétique et pauvre en protéine ; une plus faible ingestion des fourrages liée à la réduction des bactéries cellulolytiques et hémicellulolytiques ; et l'absence de certains acides animés essentiels au bon fonctionnement des microorganismes du caecum (Baumont et al., 2009). Malheureusement, ce régime est à l'image de plusieurs rations alimentaires distribuées en Afrique aux animaux (ovins, caprins et volailles traditionnelles) en raison de la rareté ou la cherté des sources de protéines indispensables à la croissance optimale (Kenfack et al., 2006). 
N.D.V. KOUAKOU et Y.B.S. BROU/Int. J. Biol. Chem. Sci. 10(3): 1199-1209, 2016

Tableau 2 : Paramètres d'ingestion volontaire journalière de la matière sèche (IVJMS) des cobayes au cours de la période de gestation et de lactation

\begin{tabular}{|c|c|c|c|c|c|c|c|c|c|c|c|c|}
\hline \multirow[b]{3}{*}{ Aliments } & \multicolumn{12}{|c|}{ Régimes alimentaires } \\
\hline & \multicolumn{3}{|c|}{ PEF } & \multicolumn{3}{|c|}{ PEM } & \multicolumn{3}{|c|}{ PEMF } & \multirow[b]{2}{*}{$F p$} & \multicolumn{2}{|c|}{ PG } \\
\hline & Pan & Euph & $F p$ & Pan & Euph & Maïs & Pan & Euph & Maïs & & Pan & Gran \\
\hline IVJMS des aliments (g) & $43 \pm 10 \mathrm{a}$ & $18 \pm 4 \mathrm{a}$ & $2 \pm 1 \mathrm{a}$ & $38 \pm 10 b$ & $11 \pm 4 b$ & $11 \pm 3 \mathrm{a}$ & $41 \pm 11 \mathrm{a}$ & $13 \pm 4 c$ & $8 \pm 4 b$ & $1 \pm 1 b$ & $56 \pm 16 \mathrm{c}$ & $21 \pm 6$ \\
\hline IVJMS des régimes (g) & \multicolumn{2}{|c|}{$63 \pm 12 a$} & & \multicolumn{2}{|c|}{$59 \pm 10 \mathrm{a}$} & & \multicolumn{3}{|c|}{$63 \pm 11 a$} & & \multicolumn{2}{|c|}{$77 \pm 17 b$} \\
\hline Protéine (g MS) & \multicolumn{2}{|c|}{$8,3 \pm 1,3$} & & \multicolumn{2}{|c|}{$6,5 \pm 1,0$} & & \multicolumn{3}{|c|}{$7,6 \pm 1,3$} & & \multicolumn{2}{|c|}{$8,4 \pm 1,8$} \\
\hline EM total $(\mathrm{Kj})$ & \multicolumn{2}{|c|}{$335,4 \pm 56,6$} & & \multicolumn{2}{|c|}{$375,0 \pm 59,8$} & & \multicolumn{3}{|c|}{$385,6 \pm 78,3$} & & \multicolumn{2}{|c|}{$420,7 \pm 87,4$} \\
\hline \multirow[t]{2}{*}{ EM/Prot. (Kj/g MS) } & \multicolumn{2}{|c|}{40,5} & & \multicolumn{3}{|c|}{58,1} & \multicolumn{3}{|c|}{50,4} & & \multicolumn{2}{|c|}{50,1} \\
\hline & Pan & Euph & $F p$ & Pan & Euph & Maïs & Pan & Euph & Maïs & $F p$ & Pan & Gran \\
\hline IVJMS des aliments (g) & $55 \pm 10 \mathrm{a}$ & $26 \pm 4 a$ & $1 \pm 1 \mathrm{a}$ & $52 \pm 9 a$ & $9 \pm 3 b$ & $14 \pm 3 \mathrm{a}$ & $58 \pm 13 a$ & $16 \pm 6 c$ & $15 \pm 6 a$ & $1 \pm 1 \mathrm{a}$ & $79 \pm 24 b$ & $33 \pm 13$ \\
\hline IVJMS des régimes (g) & \multicolumn{3}{|c|}{$82 \pm 12 \mathrm{ab}$} & \multicolumn{3}{|c|}{$74 \pm 10 a$} & \multicolumn{3}{|c|}{$91 \pm 20 \mathrm{ab}$} & & \multicolumn{2}{|c|}{$111 \pm 34 b$} \\
\hline EM total $(\mathrm{Kj})$ & \multicolumn{3}{|c|}{$432,3 \pm 61,6$} & \multicolumn{3}{|c|}{$456,9 \pm 65,7$} & \multicolumn{3}{|c|}{$581,5 \pm 143,7$} & & \multicolumn{2}{|c|}{$616,1 \pm 198,2$} \\
\hline Protéine (g MS) & \multicolumn{3}{|c|}{$10,2 \pm 1,5$} & \multicolumn{3}{|c|}{$7,8 \pm 1,0$} & \multicolumn{3}{|c|}{$10,6 \pm 2,5$} & & \multicolumn{2}{|c|}{$12,1 \pm 3,6$} \\
\hline EM/Prot. (Kj/g MS) & & 2,6 & & 58 & & & & 54,7 & & & 51, & \\
\hline
\end{tabular}


Tableau 3: Coefficients d'utilisation digestive apparent (CUDa) des régimes étudiés au cours de l'essai.

\begin{tabular}{|c|c|c|c|c|}
\hline \multirow{2}{*}{ CUDa (\%) } & \multicolumn{4}{|c|}{ Régimes alimentaires } \\
\hline & PEF & PEM & PEMF & PG \\
\hline Matière sèche & $55,1 \pm 11,4 \mathrm{~b}$ & $70,3 \pm 8,7 \mathrm{a}$ & $60,8 \pm 13,2 b$ & $52,2 \pm 17,4 b$ \\
\hline Matière organique & $54,4 \pm 11,7 \mathrm{bc}$ & $70,7 \pm 8,6 a$ & $60,9 \pm 13,4 c$ & $51,7 \pm 17,7 b$ \\
\hline Matière azotée totale & $90,7 \pm 1,9 b$ & $88,6 \pm 3,5 a$ & $91,1 \pm 3,0 b$ & $91,6 \pm 2,6 b$ \\
\hline $\begin{array}{l}\text { Les moyennes portant } 1 \\
\text { PEM : Panicum maxim } \\
\text { PEF : Panicum maximu } \\
\text { PEMF : Panicum maxin } \\
\text { PG : Panicum maximun }\end{array}$ & $\begin{array}{l}\mathrm{s} \text { minuscules sur lic } \\
\text { a heterophylla ass } \\
\text { heterophylla asso } \\
\text { ia heterophylla as } \\
\text { nulé pour lapin. }\end{array}$ & $\begin{array}{l}\text { me ligne sont } \mathrm{s} \\
\text { au maïs-grain } \\
\text { à la farine de } \mathrm{p} \\
\text { és au maïs-grai }\end{array}$ & $\begin{array}{l}\text { iquement identic } \\
\text { la farine de poi }\end{array}$ & \\
\hline
\end{tabular}

Tableau 4: Paramètres de la reproduction et de croissance moyenne journalière (GMQ) des animaux soumis aux régimes étudiés au cours de l'essai.

\begin{tabular}{|c|c|c|c|c|c|}
\hline \multirow{2}{*}{ Animaux } & \multirow{2}{*}{ Paramètres } & \multicolumn{3}{|c|}{ Régimes alimentaires } & \multirow[b]{2}{*}{ PG } \\
\hline & & PEF & PEM & PEMF & \\
\hline \multirow{7}{*}{ Gestation } & $\begin{array}{l}\text { Nombre de femelles } \\
\text { mises en lutte }\end{array}$ & 6 & 6 & 6 & 6 \\
\hline & GMQ (g.j $\left.{ }^{-1}\right)$ & $4 \pm 1 b$ & $2 \pm 0 \mathrm{a}$ & $4 \pm 1 b$ & $6 \pm 1 c$ \\
\hline & Taux de fécondité(1) & 100,0 & 83,3 & 166,7 & 200 \\
\hline & Poids des portées (g) & $147 \pm 41 \mathrm{a}$ & $121 \pm 28 \mathrm{a}$ & $176 \pm 57 \mathrm{a}$ & $208 \pm 80 \mathrm{a}$ \\
\hline & Taille des portées & $1,5 \pm 0,6 \mathrm{a}$ & $1,7 \pm 0,6 \mathrm{a}$ & $2,0 \pm 0,7 \mathrm{a}$ & $2,0 \pm 0,7 \mathrm{a}$ \\
\hline & P.m.m.b. ${ }^{(2)}$ & $722,1 \pm 80,3 \mathrm{a}$ & $655,7 \pm 50,0 \mathrm{a}$ & $672,3 \pm 101,4 a$ & $834 \pm 107 b$ \\
\hline & $\begin{array}{l}\text { P.m.n. des jeunes } \\
\text { cobayes }^{(3)}\end{array}$ & $98 \pm 17 b$ & $71 \pm 14 a$ & $88 \pm 18 \mathrm{ab}$ & $104 \pm 6 b$ \\
\hline \multirow{4}{*}{ Lactation } & $\begin{array}{l}\text { P.m.s. des jeunes } \\
\text { cobayes }^{(4)}\end{array}$ & $225 \pm 46 a b$ & $186 \pm 36 a$ & $227 \pm 46 \mathrm{ab}$ & $268 \pm 27 b$ \\
\hline & P.m.r.s. ${ }^{(5)}$ & $532,3 \pm 88,4 a$ & $533,5 \pm 53,2 \mathrm{a}$ & $710,3 \pm 24,1 \mathrm{a}$ & $750 \pm 100 \mathrm{a}$ \\
\hline & GMQ $\left(g \cdot j^{-1}\right)^{(6)}$ & $6 \pm 1 \mathrm{ab}$ & $5 \pm 1 \mathrm{a}$ & $7 \pm 2 \mathrm{ab}$ & $8 \pm 1 b$ \\
\hline & $\operatorname{GMQ}\left(\mathrm{g} \cdot \mathrm{j}^{-1}\right)^{(7)}$ & $-3 \pm 1 \mathrm{a}$ & $-3 \pm 1 \mathrm{a}$ & $-2 \pm 2 \mathrm{a}$ & $-4 \pm 1 \mathrm{a}$ \\
\hline
\end{tabular}

$\mathrm{a}, \mathrm{b}$ : les moyennes portant les mêmes lettres sur la même ligne ne sont pas statistiquement différentes.

PEM : Panicum maximum et Euphorbia heterophylla associé au maïs-grain ; PEF : Panicum maximum et Euphorbia heterophylla associé à la farine de poisson ; PEMF : Panicum maximum et Euphorbia heterophylla associé au maïs-grain et à la farine de poisson ; PG : Panicum maximum associé au granulé pour lapin.

(1) Taux de fécondité : nombre de petits nés/Nombre de femelles mises en reproduction ; (2) P.m.m.b. : poids moyen des reproductrices à la mise bas ; (3) P.m.n. : poids moyen des cobayes à la naissance ; (4) P.m.s. : poids moyen des jeunes cobayes au sevrage ; (5) P.m.r.s. : poids moyen des reproductrices au sevrage ; (6) Gain Moyen Quotidien GMQ (g.j ${ }^{-1}$ )= (Pms-Pmn)/Durée de la période (21 jours) ; (7) Gain Moyen Quotidien GMQ $\left(\mathrm{g} . \mathrm{j}^{-1}\right)=($ Pmrs-Pmrmb)/Durée de la période $(21$ jours). 
Tableau 5 : Gain de poids des femelles suitées (en g) et coûts (en F CFA) des aliments expérimentaux distribués frais non séchés.

\begin{tabular}{|c|c|c|c|c|}
\hline \multirow{2}{*}{ Cycle de reproduction } & \multicolumn{4}{|c|}{ Régimes alimentaires } \\
\hline & PEF & PEM & PEMF & PG \\
\hline Gain de poids de femelles suitées & $384 \pm 63 a b$ & $235 \pm 25 b$ & $479 \pm 59 \mathrm{~b}$ & $573 \pm 158 b$ \\
\hline Coût de Panicum maximum & 158,7 & 142,9 & 155,7 & 212,5 \\
\hline Coût de Euphorbia heterophylla & 100,3 & 53,3 & 69,2 & 0,0 \\
\hline Coût de maïs-grain & 0,0 & 148,5 & 122,1 & 0,0 \\
\hline Coût de farine de poisson & 116,1 & 0,0 & 65,6 & 0,0 \\
\hline Coût du granulé pour lapin & 0,0 & 0,0 & 0,0 & 1032,8 \\
\hline Coût total de l'alimentation & 375,1 & 344,7 & 412,7 & 1245,3 \\
\hline Coût total de l'alimentation ${ }^{(3)}$ & 116,1 & 148,5 & 187,7 & 1032,8 \\
\hline $\begin{array}{l}\text { Coût relatif de l'alimentation par } 100 \\
\text { g de gain poids des femelles suitées }\end{array}$ & 97,6 & 146,6 & 86,1 & 217,3 \\
\hline 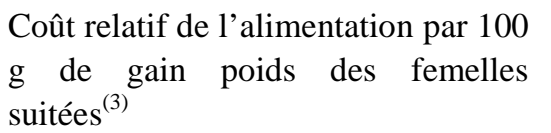 & 30,2 & 63,1 & 39,2 & 180,2 \\
\hline $\begin{array}{l}\text { Les valeurs portant les mêmes lettres en exposant } \\
\text { (1) L'acquisition et la distribution de Panicum mo } \\
\text { CFA/kg; } \\
\text { (2) L'acquisition du granulé pour lapin, du maïs-g } \\
\text { 650 F CFA/kg; } \\
\text { (3) Le coût de l'alimentation ne tient pas compt } \\
\text { heterophylla frais non séchés. }\end{array}$ & $\begin{array}{l}\text { tême ligne } \\
\text { et de Euph } \\
\text { de la farine }\end{array}$ & son ont ét & $\begin{array}{l}\text { qques. } \\
\text { on séché } \\
\text { es respec }\end{array}$ & $\begin{array}{l}\text { estimées à } 8 \mathrm{~F} \\
\text { dt à } 250,120 \mathrm{e} \\
\text { de Euphorbic }\end{array}$ \\
\hline
\end{tabular}

L'efficacité comparatif du régime $\mathrm{PEF}$ par rapport au régime PEM est due à : un meilleur équilibre protéine/Energie ; un niveau d'ingestion de la farine de poisson qui aurait accentué la prolifération et le dynamisme des bactéries cellulolytiques et hémicellulolytiques induisant ainsi une meilleure ingestion de Panicum maximum; et une augmentation significative du niveau d'ingestion de Euphorbia heterophylla (Kouakou et al., 2010). Aussi, dans un milieu rural africain, où la récolte de fourrage ne peut être facturée car s'inscrivant dans les activités quotidiennes d'entretien des parcelles agricoles, ce régime est-il le plus économique. Toutefois, malgré ces avantages comparatifs, la concurrence alimentaire sur la farine de poisson pourrait être une contrainte. Dans ces conditions, des essais portant sur la substitution de cette farine par des asticots produits sur des substrats constitués de déchets et abats de poissons devraient être envisagés.

Le régime PEMF est le plus équilibré en énergie et en protéine d'origine animale, comblant ainsi les déficits observés dans les deux autres régimes (PEF et PEM) et induisant des performances zootechniques proches de celui du régime témoin ( $\mathrm{PG}$ ). L'avantage de PEMF, un régime fourrager avec un complément glucidique et protéique contrairement à PEM, est qu'il induit une diminution de l'activité cellulolytique, la plus faible possible de sorte à ne pas créer une 
chute du $\mathrm{pH}$ dans le caecum consécutive à la production rapide d'AGV et une baisse de l'activité cellulolytique (Kouakou et al., 2010). Toutefois, à l'instar de PG, PEMF paraît moins économique s'il était produit en milieu rural.

Les croissances journalières des trois régimes alternatifs au régime témoin (PG) sont supérieures à celles obtenues suite à la distribution de Euphorbia heterophylla associé à $P$. maximum chez le cobaye $(4,1$ g.j ${ }^{1}$ ) (Kouakou et al., 2015a), de la légumineuse Desmodium intortum associé à Pennisetum purpureum durant la lactation chez le cobaye (3,5 g.j ${ }^{-1}$ ) (Tchoumboué et al., 2001) et de Tithonia diversifolia associé à Pennisetum purpuruem (2,5 g.j j $^{-1}$ ) (Noumbissi et al., 2014). Les résultats obtenus dans cette étude montrent que le régime constitué de Panicum maximum, Euphorbia heterophylla et de farine de poisson semble être en milieu rural, un compromis idéal à mieux étudier car il présente un avantage comparatif certain.

\section{Conclusion}

En caviaculture, une alimentation de qualité à moindre coût en milieu rural est possible en utilisant Panicum maximum, Euphorbia heterophylla, et de la farine de poisson afin d'améliorer la productivité des cobayes pour le bonheur des éleveurs. Cependant, compte tenu des contraintes économiques pour l'acquisition de la farine de poisson, la recherche d'une autre source protéique d'origine animale telle que les farines d'asticots produits sur des substrats et abats de poisson mériterait d'être envisagée.

\section{CONFLIT INTERETS}

Les auteurs déclarent qu'ils n'ont aucun conflit d'intérêts.

\section{CONTRIBUTIONS DES AUTEURS}

NDVK a contribué de manière significative à la conception et à la mise en place du plan de travail. YBSB et NDVK ont contribué de manière significative à la mise en place du plan de travail, à la collecte des données et à l'analyse et interprétation des résultats. Les deux auteurs suscités ont participé à l'organisation des idées, à la révision du contenu intellectuel du document et sont à mesure d'en défendre individuellement le contenu.

\section{REMERCIEMENTS}

Les coauteurs tiennent à remercier toutes les personnes physiques ou morales qui ont contribué de près ou de loin à l'obtention des présents résultats.

\section{RÉFÉRENCES}

AOAC. 2006. Official Methods of Analysis $\left(18^{\text {th }}\right.$ edn). Association of Official Analytical Chemists. Association of Analytical Communities: Gaithersburg, MD, USA,

Baumont R, Aufrère J, Meschy F. 2009. La valeur alimentaire des fourrages : rôle des pratiques de culture, de récolte et de conservation. Fourrages, 198: 153173.

Bindelle J, Buldgen A, Boudry C, Leterme P. 2007. Effect of inoculum and pepsinpancretain hydrolysis on fibre fermentation measured by the gas production technique in pigs. Anim. Feed Sci. Technol., 132: 111-122. Doi:10.1016/j.anifeedsci.2006.03.009

Bindelle J, Kinsama A, Picron P, Umba di M'Balu J, Kindele E, Buldgen A. 2009. Nutritive value of unconventional fibrous ingrdients fed to guinea pigs in the Democratic Republic of Congo. Trop. Anim. Health Prod., 41: 17311740. DOI: $10.1007 /$ s11250-009-93721

Franz R, Kreuzer M, Hummel J, Hatt J-M, Clauss M. 2011. Intake, selection, 
digesta retention, digestion and gut fill of two coprophageous species, rabbits (Oryctolagus cuniculus) and guinea pigs (Cavia porcellus), on a hay-only diet. J. Anim. Physiol. Anim. Nutr., 95: 564-570. DOI: $10.1111 / \mathrm{j} .1439$ 0396.2010.01084.x

Fuss S. 2002. Physiologie et pathologie digestive chez le cobaye domestique (Cavia porcellus). Docteur Vétérinaire Thèse d'Etat, Ecole Nationale Vétérinaire de Toulouse, p. 212.

Jarrige R, Grenet E, Demarquilly C, Besle JM. 1995. Les constituants de l'appareil végétatif des plantes fouragères. In Nutrition des Ruminants Domestiques, Ingestion et Digestion, Jarrige R, Ruckebusch Y, Demarquilly C, Farce M-H, Journet M (eds). Inra Editions: Paris; 25-81.

Kenfack A, Tchoumboué J, Kamtchouing P, Ngoula F. 2006. Effets de la substitution par l'arachide fourragère (Arachis glabrata) de l'herbe à éléphant (Pennisetum purpureum) sur le nombre d'ovulations et les mortalités prénatales chez le cobaye (Cavia porcellus L.) adulte. Tropicultura, 24(3): 143-146.

Kouakou NDV, Thys E, Assidjo EN, Grongnet JF. 2010. Ingestion et digestibilité in vivo du Panicum maximum associé à trois compléments: tourteau de Jatropha curcas, tourteau de coton (Gossypium hirsutum) et Euphorbia heterophylla chez le cobaye (Cavia porcellus L.). Tropicultura, 28(3): 173177.

Kouakou NDV, Thys E, Danho M, Assidjo EN, Grongnet JF. 2012. Effet du Panicum maximum sur la productivité des femelles primipares durant le cycle de reproduction chez le cobaye (Cavia porcellus L.). Tropicultura, 30(1): 142167.
Kouakou NDV, Thys E, Yapi YM, Assidjo EN, Marnet PG, Grongnet JF. 2015a. Effets de Panicum maximum associé à Euphorbia heterophylla sur la productivité des femelles durant le cycle de reproduction chez le cobaye (Cavia porcellus L.). Tropicultura, 33(4): 277-287.

Kouakou NDV, Angbo-Kouakou CEM, Assidjo NE, Grongnet JF. 2015 b. Stratégies incitatives à la pratique de l'élevage des cobayes (Cavia porcellus L.) en Côte d'Ivoire. Int. J. Biol. Chem. Sci., 9(2): 664-678. DOI: http://dx.doi.org/10.4314/ijbcs.v9i2.8.

Miégoué E, Tendonkeng F, Lemoufouet J, Noumbissi MNB, Mweugang NN, Zougou GT, Boukila B, Nkouadjio MF et Pamo ET. 2016. Croissance présevrage des cobayes nourris au Panicum maximum supplémenté avec une ration contenant Arachis glabrata, Calliandra calothyrsus ou Desmodium intortum. Int. J. Biol. Chem. Sci., 10(1): 313-325. DOI http://dx.doi.org/10.4314/ijbcs.v10i1.24 Mweugang NN, Tendonkeng F, Miégoué E, Matumuini FEN., Zougou GT, Fonteh FA, Boukila B et Pamo ET. 2016. Effets de l'inclusion de feuilles de manioc (Manihot esculenta Crantz) dans la ration sur les performances de reproduction du cobaye (Cavia porcellus L.) local camerounais. Int. J. Biol. Chem. Sci., 10(1): 269-280. DOI : http://dx.doi.org/10.4314/ijbcs.v10i1.21 Noumbissi MNB, Tendonkeng F, Zougou TG, Pamo Tendonkeng E. 2014. Effet de différents niveaux de supplémentation de feuilles de Tithonia diversifolia (Hemsl.) A. Gray sur l'ingestion et la digestibilité in vivo de Pennisetum purpureum K. Schum. chez le cobaye (Cavia porcellus L.). Tropicultura, 


$$
\text { 32(3): 138-146. }
$$

Sakaguchi E, Itoh I, Kohno T, Ohshima S, Mizutani K. 1997. Fiber digestion and weight gain in guinea pigs fed diets containing different fiber sources. Exp. Anim., 46(4): 297-302.

Sakaguchi E. 2003. Digestive strategies of small hindgut fermenters. Anim. Sci. J., 74(5): 327-337.

STATA. 2012 Stata/IC 12.0 for windows. Lakeway Drive College Station.

Tchoumboué J, Niba AT, Kenfack A. 2001. Comparative study on the influence of supplementation with two legumes (Arachis glabrata benth and Desmodium intortum) on the reproductive and growth performance of guinea pigs (Cavia porcellus L.). Bull. Anim. Hlth. Prod. Afr., 49: 74-83.
Tedonkeng EP, Niba AT, Fonteh FA, Tedonkeng F, Kana JR, Boukila B, Tsachoung J. 2005. Effet de la supplémentation au Moringa oleifera ou aux blocs multinutritionnels sur l'évolution du poids post-partum et la croissance pré-sevrage des cobayes (Cavia porcellus L.). Livest. Res. Rural Dev., 17(4). http://www.lrrd.org/ lrrd17/4/tedo17046.htm

Yapi YM, Zongo D et Iritié BM. 2013. Effet d'une réduction simultanée des taux de fibres et de protéines brutes de la ration sur la santé et la croissance de l'aulacode. Int. J. Biol. Chem. Sci., 7(6): 2264-2274, DOI : http://dx.doi.org/10.4314/ijbcs.v7i6.7 